\title{
Non-disjunction of chromosome 13
}

\author{
Merete Bugge ${ }^{1,2, *, \dagger}$, Andrew Collins ${ }^{3, \dagger}$, Jens Michael Hertz ${ }^{4}$, Hans Eiberg ${ }^{5}$, Claes Lundsteen ${ }^{6, \$}$, \\ Carsten A. Brandt ${ }^{4,7}$, Mads Bak ${ }^{1}$, Claus Hansen ${ }^{1}$, Celia D. deLozier ${ }^{8}$, James Lespinasse ${ }^{9}$, \\ Lisbeth Tranebjaerg ${ }^{10}$, Johanne M.D. Hahnemann ${ }^{11}$, Kirsten Rasmussen ${ }^{12}$, \\ Gert Bruun-Petersen ${ }^{7}$, Laurence Duprez ${ }^{13}$, Niels Tommerup ${ }^{1}$ and Michael B. Petersen ${ }^{2,14, *}$
}

${ }^{1}$ Wilhelm Johannsen Centre for Functional Genome Research, Department of Cellular and Molecular Medicine, University of Copenhagen, Denmark, ${ }^{2}$ Department of Medical Genetics, The John F Kennedy Institute, Glostrup, Denmark, ${ }^{3}$ Human Genetics, University of Southampton, Duthie Building (808), Southampton General Hospital, Southampton SO16 6YD, England, ${ }^{4}$ Department of Clinical Genetics, Aarhus University Hospital, Aarhus, Denmark, ${ }^{5}$ Department of Cellular and Molecular Medicine, University of Copenhagen, Denmark, ${ }^{6}$ Department of Clinical Genetics, Rigshospitalet, Copenhagen, Denmark, ${ }^{7}$ Department of Clinical Genetics, County Hospital of Vejle, Denmark, ${ }^{8}$ Department of Genetics and Microbiology, University of Geneva School of Medicine, Switzerland, ${ }^{9}$ Cytogenetics Laboratory, General Hospital, Chambery Cedex, France, ${ }^{10}$ Department of Medical Genetics, University Hospital of Tromsø, Norway, ${ }^{11}$ Medical Genetics Laboratory Centre, The Kennedy Institute-National Eye Clinic, Glostrup, Denmark, ${ }^{12}$ Department of Clinical Genetics, University Hospital of Odense, Odense, Denmark, ${ }^{13}$ Laboratoire de Cytogénétique, ULB-Erasme-CHU Brygman, Hôpital ULB Erasme, Bruxelles, Belgium and ${ }^{14}$ Department of Genetics, Institute of Child Health, Athens, Greece

Received March 30, 2007; Revised and Accepted June 8, 2007

We performed a molecular study with 21 microsatellites on a sample of 82 trisomy 13 conceptuses, the largest number of cases studied to date. The parental origin was determined in every case and in $89 \%$ the extra chromosome 13 was of maternal origin with an almost equal number of maternal MI and MII errors. The latter finding is unique among human autosomal trisomies, where maternal MI (trisomies 15, 16, 21, 22) or MII (trisomy 18) errors dominate. Of the nine paternally derived cases five were of MII origin but none arose from Ml errors. There was some evidence for elevated maternal age in cases with maternal meiotic origin for liveborn infants. Maternal and paternal ages were elevated in cases with paternal meiotic origin. This is in contrast to results from a similar study of non-disjunction of trisomy 21 where paternal but not maternal age was elevated. We find clear evidence for reduced recombination in both maternal MI and MII errors and the former is associated with a significant number of tetrads (33\%) that are nullichiasmate, which do not appear to be a feature of normal chromosome 13 meiosis. This study supports the evidence for subtle chromosome-specific influences on the mechanisms that determine non-disjunction of human chromosomes, consistent with the diversity of findings for other trisomies.

\section{INTRODUCTION}

Trisomy 13, or Patau syndrome, was first described by Patau et al. (1). With prevalence rates at birth estimated between $1 / 4000$ and $1 / 29000(2,3)$, Patau syndrome is the third most common autosomal trisomy among newborns, after Down syndrome and Edwards syndrome. Trisomy 13 occurs in $\sim 2 \%$ of first trimester spontaneous abortions, where it is the fourth commonest autosomal trisomy following those of chromosomes 16, 21 and 22 (4). Clinical findings in Patau syndrome include severe mental and growth retardation, as well as varying combinations of microcephaly, ocular malformations, polydactyly, cleft lip and/or palate, omphalocele,

*To whom correspondence should be addressed at: Tel: +45 35327809; Fax: +45 35327845; Email: mb@imbg.ku.dk

The authors wish it to be known that, in their opinion, the first two authors should be regarded as joint First Authors.

†C.L. died on 10 November 2003. 
Table 1. Study population

\begin{tabular}{|c|c|c|c|c|c|}
\hline \multirow[t]{2}{*}{ Ascertainment } & \multicolumn{4}{|c|}{ Number of cases } & \multirow[t]{2}{*}{ Maternal age $($ mean $\pm S D)$} \\
\hline & Total & Female & Male & $\mathrm{M} / \mathrm{F}$ ratio & \\
\hline Prenatal advanced age & 25 & $14^{\mathrm{a}}$ & 11 & 0.8 & $38.1 \pm 2.6$ \\
\hline Spontaneous abortions & 4 & 2 & 2 & 1 & $35.8 \pm 5.3$ \\
\hline Live birth & 26 & 10 & $16^{\mathrm{b}}$ & 1.6 & $30.7 \pm 4.3$ \\
\hline Stillbirth & 2 & 1 & 1 & 1 & $34.5 \pm 3.5$ \\
\hline Total & 82 & 35 & 47 & 1.3 & $33.1 \pm 5.0$ \\
\hline
\end{tabular}

${ }^{a}$ Including one case with $46, \mathrm{XX} / 47, \mathrm{XX}+13$.

${ }^{\mathrm{b}}$ Including one case with $47, \mathrm{XY},+13 / 48, \mathrm{XYY},+13$.

Table 2. Classification of cases by parental origin and meiotic error

\begin{tabular}{lllllllll}
\hline & \multicolumn{3}{l}{ Meiotic stage of error } & & & & & \\
& MI(t) & MI(n) & MI(?) & MII(t) & MII(n) & MII(?) & PZM & Unknown meiotic stage \\
\hline Maternal & 17 & 13 & 4 & 29 & 3 & 1 & 3 & 3 \\
Paternal & 0 & 0 & 0 & 5 & 0 & 0 & 3 & 1 \\
Total & 17 & 13 & 4 & 34 & 3 & 1 & 6 & 4 \\
\hline
\end{tabular}

(t), cases with one or more transitions; (n), nulli-chiasmate cases; (?), cases where meiotic stage determined but fewer than seven markers informative about transition distribution.

cardiac and renal anomalies, leading to a $95 \%$ mortality rate within the first year of life $(2,3)$. As with the autosomal trisomies 16,18 and 21 , there is a strong association with increased maternal age (4).

Trisomy 13 occurs with either a free additional chromosome 13 or as a Robertsonian translocation involving a chromosome 13 , usually a de novo translocation $\mathrm{t}(13 ; 14)$ or $\mathrm{t}(13 ; 13)$ or as an isochromosome $\mathrm{i}(13 ; 13)$. DNA studies of the parental origin of the additional chromosome 13 in cases of free trisomy 13 have, to date, been undertaken using RFLP markers and microsatellites in a very limited number of cases: RFLP markers: 8 cases (5); microsatellites: 27 cases (6) and 19 cases (7). In those studies it was not possible to determine the parental origin in all cases, and it was only possible to determine the meiotic stage of non-disjunction in a fraction of the cases.

We investigate here a total of 82 trisomy 13 cases. This is by far the largest sample of trisomy 13 cases examined to date and enables the determination of parental origin and meiotic stage of non-disjunction of the extra chromosomes. We have also constructed non-disjunction maps for this chromosome for the first time to evaluate whether patterns of recombination are altered in the trisomic cases, as has been found in other trisomies.

\section{RESULTS}

\section{Patient populations}

The sample comprises 82 trisomy 13 conceptuses and their parents. Although 5 families came from Norway, 4 from Switzerland, 4 from France, 6 from Belgium and one from Greece, the majority of 62 were from Denmark. A total of 50 cases were obtained through routine prenatal diagnosis, along with
26 live births, four spontaneous abortions and two stillborn children (Table 1).

\section{Cytogenetic analysis}

The karyotypes of the probands are $47, \mathrm{XX},+13$ in 34 cases, $47, X Y,+13$ in 46 cases, $46, X X / 47, X X+13$ in one and $47, X Y+13 / 48, X Y Y+13$ in one case. The karyotypes of all parents were normal, except for one father who was carrier of a rob(13;14). The child of this father had a free trisomy 13 of maternal origin.

\section{Parental origin and cell division error}

Tables 2 and 3 give a breakdown of parental origin by meiotic stage. From the total of 82 cases, 73 are of maternal origin $(89 \%)$ with an almost equal number of errors in the first and second meiotic stages (MI: 34 and MII: 33), and 3 are considered to be post-zygotic mitotic (PZM) errors. The majority of the nine paternally derived cases are due to meiotic errors, as five are MII, one unknown, but most likely to be MII and three most likely to be PZM (Tables 2 and 3). In five of the six cases originating from meiotic paternal errors the probands were males and only one was a female, and the three cases due to paternal mitotic error were all males.

\section{Maternal age}

The maternal ages and maternal ages by cell division error are given in Tables 1 and 3. Nearly one-third of the conceptuses were ascertained by prenatal diagnosis because of advanced maternal age (35 years and older). Of the 26 live births, 19 were Danish and, with one exception, the mothers were all younger than 35 years at the time of conception (mean maternal 
Table 3. Maternal age and cell division error

\begin{tabular}{|c|c|c|c|c|}
\hline \multirow[t]{2}{*}{ Cell division } & \multicolumn{2}{|c|}{ All cases } & \multicolumn{2}{|c|}{$\begin{array}{l}\text { Excluding prenatal cases ascertained } \\
\text { because of advanced maternal age }\end{array}$} \\
\hline & No & $\begin{array}{l}\text { Maternal age } \\
(\text { mean }+ \text { SD) }\end{array}$ & No & $\begin{array}{l}\text { Maternal age } \\
(\text { mean }+\mathrm{SD})\end{array}$ \\
\hline All & 82 & $33.1 \pm 5.0$ & 54 & $31.1 \pm 4.1$ \\
\hline Maternal MI & 34 & $33.6 \pm 4.4$ & 23 & $31.7 \pm 3.8$ \\
\hline Maternal MII & 33 & $33.8 \pm 5.7$ & 22 & $31.2 \pm 4.7$ \\
\hline Maternal PZM & 3 & $28.0 \pm 3.6$ & 3 & $28.0 \pm 3.6$ \\
\hline Maternal MI or MII & 3 & $33.5 \pm 4.0$ & 2 & $31.5 \pm 2.1$ \\
\hline Paternal MII & 5 & $33.8 \pm 1.3$ & 3 & $33.0 \pm 0.8$ \\
\hline Paternal PZM & 3 & $32.3 \pm 6.0$ & 2 & $29.5 \pm 3.5$ \\
\hline Paternal MI or MII & 1 & 26 & & \\
\hline
\end{tabular}

age 30.5 ). We analysed maternal ages for the Danish cases, where we have detailed information about national maternal ages. The Danish cases were born in the period 1984-2004, during which the national mean maternal age for mothers in Denmark varied between 27.5 and 30.2 years (overall mean 28.9) and from 26.5 to 28.8 years (overall mean 27.9), excluding those who are 35 years or more at the time of childbirth (8). A Wilcoxon matchedpairs comparison of the 19 maternal ages with corresponding national mean ages yields $P<0.0874$ and $P<0.00823$ when national means exclude those aged $>35$. There is therefore a suggestion that the mean maternal age is still elevated when the cases ascertained because of advanced maternal age are excluded.

The mean maternal age is elevated for spontaneous abortions and stillbirths, but these groups represent only six cases in total. There is no difference in the mean maternal age for mothers of maternal meiosis I and II errors. The parents of the six cases with paternal meiotic errors have advanced parental age as the mean maternal age is 32.5 and the mean paternal age is 39.0 years.

\section{The frequency of nullichiasmate tetrads}

The frequency of nullichiasmate tetrads was examined in both normal meiosis and non-disjunction (Table 4). There is no evidence for nullichiasmate tetrads in both normal chromosome 13 meiosis and MII trisomy cases $\left(\chi^{2} 0-1.42\right)$, but strong evidence for nullichiasmate tetrads in $33 \%$ of maternal MI cases $\left(\chi^{2} 6.74, P<0.01\right)$.

\section{Non-disjunction maps}

Figure 1 compares non-disjunction maps from the maternal cases with the standard linkage map. The standard map comprises 21 markers between HECH1 and D13S285 and spans $156.04 \mathrm{cM}$. Both non-disjunction maps are shorter than the standard map (MI: $132.65 \mathrm{cM} \pm 19.45$; MII: $125.23 \mathrm{cM} \pm 12.74)$. Relative to the standard map differences in the slopes of the non-disjunction maps suggest regional distortions in the recombination rate. Both MI and MII maps show increased recombination close to the centromere (the first $\sim 0-20 \mathrm{cM}$ on the standard map). The central portion of $13 \mathrm{q}$ shows some regions with markedly reduced recombination in both MI and MII, although MI shows some evidence for locally elevated rates at around the $50 \mathrm{cM}$ point on the standard map. The maps provide compelling evidence for a distorted recombination pattern in both maternal MI and MII non-disjunction.

\section{DISCUSSION}

The additional chromosome 13 arose from maternal nondisjunction in $89 \%$ of the cases. This is consistent with results found in previous non-disjunction studies of trisomy 13 (Table 5). The distribution of maternal MI and MII errors shows an equal number of the two (Tables 2 and 3). This finding has not been reported in previous studies of this chromosome, as the authors were not able to distinguish between meiosis I and II errors in the majority of cases, because chromosome 13 was poorly mapped in the pericentromeric region. We therefore constructed PCR primers around a (CA)n repeat situated very close to the centromere (HECH1) and using this and nearby concordant markers, the meiotic stage was assigned in all but four cases (Table 2).

The finding of approximately equal numbers of both meiotic stage errors in maternal meiosis is unique among autosomal trisomies, as maternal MI errors are dominant in trisomy 16 (9), 21 (10) and 22 (6), and maternal MII errors dominate in trisomy 18 (11) (Table 6). However, Lamb et al. (12) indicated that some trisomy 21 cases with apparent maternal meiosis II errors could reflect a precipitating event in meiosis I. This is recognized by increased recombination in the pericentromeric region evident in the maternal MII map and the possibility that bivalents with proximal exchanges become 'entangled' and are therefore misclassified as MII errors. For chromosome 13, we have shown increased recombination in the pericentromeric region in both maternal MI and MII maps. It is possible that the same mechanism might take place in trisomy 13 in which case the relative proportions of MI and MII errors cannot be known with certainty.

The paternal meiotic error rate of $11 \%$ is higher than in studies of trisomy 16,18 and 21 , where the paternal error rate is 0,6 and $7 \%$, respectively, (Table 6). No case arose through paternal MI non-disjunction (Tables 2 and 3). This result contrasts with trisomy 21 , where one-third of the paternal meiotic errors are MI and two-thirds are MII errors, but the result is similar to the results of studies of the other acrocentric trisomies for chromosomes 14, 15 and 22 (6), although samples sizes investigated in these trisomies were very small (Table 6).

In Denmark an invasive prenatal procedure was offered to all pregnant women aged 35 years or more at the time of conception of pregnancy until the year 2004. The uptake was high for this group, especially in the early 1990s (13). In spite of this we have suggestive evidence that maternal age is somewhat elevated for the 19 Danish live births. We also find advanced maternal and paternal age in cases of paternal meiotic origin (Table 3 ). This has been observed in nondisjunction studies of trisomy 13,14, 15 and 22 (6), but is in contrast to results from trisomy 21 studies, where the maternal age was significantly lower in cases of paternal meiotic origin than of maternal meiotic origin (13), and in a study of $47, \mathrm{XXY}$ and $47, \mathrm{XXX}$ where there was no evidence of increased paternal age in the aneuploids of paternal origin (14). In the cases due to paternal non-disjunction there is an 
Table 4. The numbers of cross-overs and transitions in chromosome 13 meiosis

\begin{tabular}{lllllllllll}
\hline Data & $q 0$ & $\chi^{2}(q 0=0)$ & $n 0$ & $n 1$ & $n 2$ & $n 3$ & $n 4$ & $n 5$ & Total & Reference source \\
\hline Pat. cross. & 0.07 & 0.46 & 29 & 36 & 13 & 0 & 0 & 0 & 78 & $(11)$ \\
Pat. MII & 0.00 & 0.00 & 0 & 2 & 1 & 1 & 1 & 0 & 5 & 78 \\
Mat. cross. & 0.12 & 1.42 & 21 & 27 & 21 & 9 & 0 & 0 & $(11)$ \\
Mat. MI & 0.33 & $6.74^{*}$ & 13 & 3 & 7 & 4 & 3 & 0 & 30 & \\
Mat. MII & 0.09 & 0.00 & 3 & 11 & 7 & 7 & 3 & 1 & 32 & \\
\hline
\end{tabular}

cross., cross-over distribution in normal meiosis from CEPH families; $q 0$, frequency of nulli-chiasmate tetrads; $n 0-n 5=$ counts of cross-overs and transitions.

${ }^{*} P<0.01$ excludes cases with fewer than seven markers informative.

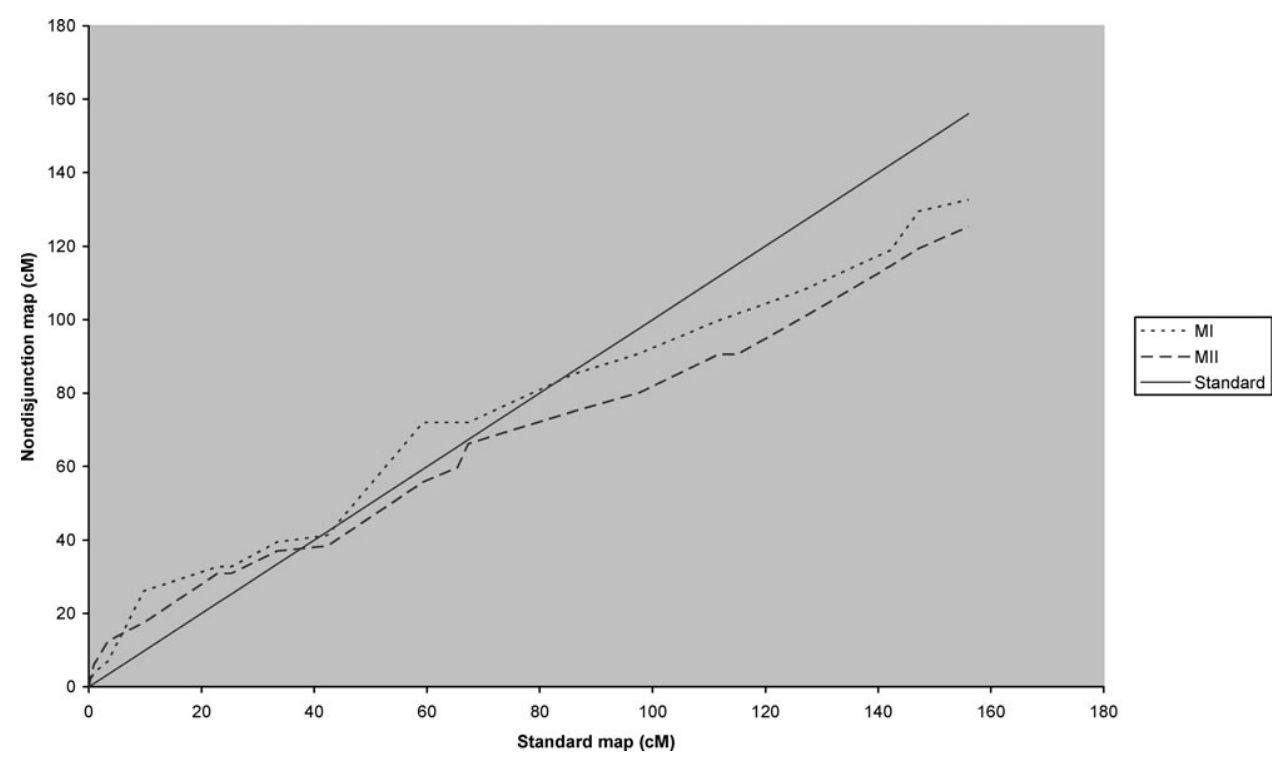

Figure 1. Maternal non-disjunction maps of chromosome 13. The figure plots meiosis I and II maternal non-disjunction maps against the standard linkage map on the $X$-axis. Increases and decreases in slope relative to the standard map indicate, respectively, increased and reduced recombination rates in the nondisjunction maps.

excess of males. A similar observation has been described in a study of paternal non-disjunction of trisomy 21 , where 21 of 36 probands were males (15).

Examination of the maternal MI non-disjunction map indicates reduced recombination compared with the standard map ( $\sim 85 \%$ of the overall length). This in part reflects a high number of nullichiasmate tetrads $(33 \%)$ which do not appear to be a feature of normal chromosome disjunction or tetrads giving rise to maternal MII errors. Interestingly, Tease et al. (16), examining MLH1 foci at the pachytene stage, observed no (presumed non-cross-over) oocytes lacking foci for chromosome 13, except in abnormal cells which showed chromosome pairing errors. The authors suggested that these abnormal fetal oocytes were the source of maternal nondisjunction errors. A similar finding of nullichiasmate tetrads has been reported previously, for example, Robinson et al. (17) estimated that $21 \%$ of tetrads leading to maternal MI nondisjunction were nulli-chiasmate on chromosome 15 .

Although the overall recombination rate is lower than the standard map across the chromosome (Fig. 1), there is a suggestion of increased intensity of recombination in the centromeric region. Some evidence for increased centromeric recombination was previously reported in chromosome 21 maternal meiosis II (12). Chromosome 18 non-disjunction is associated with even greater suppression of recombination as the maternal MI map is only $62 \%$ of the length of the standard map (11). Other chromosomes show the same features with varying degrees of intensity. The maternal MI map is 46 and $75 \%$ of the standard map length for chromosomes 21 and 16, respectively $(9,18)$. Chromosome 15 shows reduced recombination in maternal MI non-disjunction, especially on proximal 15q (19).

While there is some consistency across chromosomes with suppressed recombination in maternal MI errors this is less true for maternal MII errors. We observe an overall suppression of recombination, similar to that observed for MI but apparently more uniform along the chromosome length (Fig. 1). The overall length is $80 \%$ that of the standard map. For trisomy 21 map length is $1.46+/-0.11$ times the standard map, mostly the result of increased proximal recombination (18). Although MacDonald et al. (14) reported an overall decrease in recombination in maternal MII X chromosome trisomy there is little published evidence for suppression of recombination associated with maternal MII errors. 
Table 5. The present sample and published trisomy 13 cases classified by DNA analysis

\begin{tabular}{|c|c|c|c|c|c|c|c|c|c|}
\hline & \multirow[t]{2}{*}{ Number of cases } & \multicolumn{3}{|c|}{ Maternal error } & \multirow[t]{2}{*}{ Maternal Mitotic } & \multicolumn{3}{|c|}{ Paternal error } & \multirow[t]{2}{*}{ Paternal mitotic } \\
\hline & & MI & MII & MI/MII & & MI & MII & MI/MII & \\
\hline Hassold et al. (5) & 7 & 3 & 2 & 0 & 0 & 2 & 0 & 0 & 0 \\
\hline Robinson et al. (7) & 19 & 0 & 0 & 15 & 2 & 0 & 0 & 0 & 2 \\
\hline Present study & 82 & 34 & 33 & 3 & 3 & 0 & 5 & 1 & 3 \\
\hline Total & 130 & 41 & 35 & 33 & 6 & 3 & 6 & 1 & 5 \\
\hline
\end{tabular}

${ }^{\mathrm{a}} 27$ cases in total, but three cases were already described in Hassold et al. (5), and in two cases the parental origin was unknown.

Table 6. Parental origin and meiotic error of different autosomal trisomies

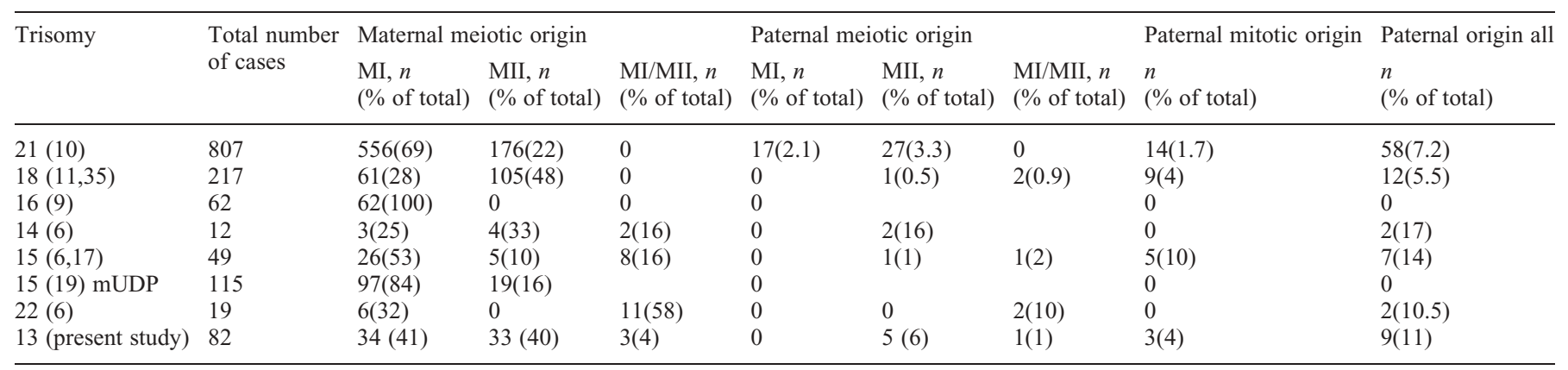

Percentages in brackets. Cases of maternal mitotic postzygotic origin are excluded.

mUPD, ascertained as maternal uniparental disomy for chromosome 15.

However, for chromosome 18 a slightly, but not significantly, reduced map length in maternal MII errors was observed (11). The possibility that at least a proportion of apparent MII errors reflect a precipitating even in MI, following Lamb et al. (12), would of course, have implications for the interpretation of these maps.

For the maternal MI and MII samples we find no evidence for a relationship between increasing number of transitions and mean maternal age as reported for trisomy 15 by Robinson et al. (19) (data not shown).

Chromosome 13 non-disjunction arises mostly through maternal meiotic errors, consistent with other trisomies, although, unusually there are equal numbers of MI and MII stage errors. There is strong evidence for suppressed recombination arising, at least in part, from nullichiasmate tetrads in MI as has been observed in other trisomies. There is less evidence for suppression of recombination associated with MII stage errors in other trisomies but it is observed for trisomy 13. However, elevated pericentromeric recombination supports the possibility that a proportion of the MII cases arose due to an error at MI. Difficulties of interpretation such as this may contribute to some of the differences in patterns of non-disjunction observed between chromosomes. One consistent finding for human trisomy, supported by our study, is that of altered recombination but establishing cause and effect is challenging. We conclude that while there are shared mechanisms there are subtle chromosome-specific differences in patterns of chromosome non-disjunction manifested as differences in recombination and variations in the meiotic stage associated with the errors.

\section{MATERIALS AND METHODS}

\section{DNA analysis}

DNA from the probands was extracted from cultured chorionic villi, amniocytes, blood, cultured fetal tissues or skin fibroblasts, and in two cases from paraffin embedded tissue by routine methods. Parental DNA was extracted from EDTA-anticoagulated blood by routine methods. In one case no sample was available from the mother. Twenty-one DNA polymorphisms were detected after PCR amplification of genomic DNA. End-labeling of primer, PCR amplification conditions, PAGE of the amplification products and autoradiography were performed according to protocols described elsewhere (20). The microsatellites used were the $(\mathrm{CA})_{\mathrm{n}}$ repeats at the following loci: D13S115 and D13S141 (21); D13S175, D13S171, D13S218, D13S155, D13S176, D13S156, D13S170, D13S154 and D13S173 (22), D13S71 (23), D13S118 and D13S120 (24), D13S1316, D13S217, D13S174, D13S1265, D13S285 (25) and the (TG) ${ }_{n}$ repeat of the human fms-related tyrosine kinase gene FLT1 (26).

The microsatellite HECH 1: In order to resolve potential cross-over events close to the centromere, the pericentromeric region on chromosome $13 q$ was scanned for repetitive regions suitable for microsatellite analysis. Primers were designed using Oligo software (Moleculart Biology Insights, West Cascade, CO, USA). A single region flanked by primers mb1A: $5^{\prime}$-TTGCAGCATTTTATATAAGATGTTT-3' and mb1B: 5'-TGTTTGAACTGAAGTTTTAAGGAT-3' amplifying a 217 bp fragment, including a polymorphic 
CA-dinucleotide repeat (rs10551654) was chosen for further analysis.

\section{Classification of cases}

The parental origin of the three chromosomes was determined by scoring the polymorphic alleles, when three different alleles were present in the proband, and by dosage analysis scored by visual comparison, when two different alleles were present in the proband (27). The meiotic stage of nondisjunction was determined on the basis of reduction (or not) to homozygosity at the pericentromeric $13 \mathrm{q}$ markers HECH1, D13S1316 or D13S141, using the methods of Chakravarti and Slaugenhaupt (28) and Chakravarti (29). The HECH1 centromere marker was informative in most cases but in a small number of cases the two most proximal markers had to be used for classification. The three markers span $0.92 \mathrm{cM}$ on the standard map and were concordant throughout, except in one case where there was a transition between D13S1316 and D13S141, raising the possibility that a small number of cases, in the subset not informative at HECH1, were misclassified.

Distinguishing PZM cases and any nulli-chiasmate MII cases, should they arise, is not possible. In the sample there are three paternal PZM cases, one of which is a mosaic. As there is no evidence to suggest there is a sex difference in the numbers of PZM cases we assumed equal numbers of paternal and maternal PZM, as assumed for trisomy 18 (11). We observed six maternal nulli-chiasmate MII or PZM cases. One was a mosaic, which we classified as PZM. As there is evidence (30) that maternal age is not advanced in PZM cases, the two other cases with young mothers, both $<30$ years, were also classified as PZM. The remaining three ambiguous maternal cases were tentatively classified as nullichiasmate MII.

\section{Chiasma distribution}

Following Bugge et al. (11), we re-constructed the chiasma distribution (Table 4) using the program Exchange (Available on request from Andrew Collins). This enables comparison of the frequency of nullichiasmate tetrads in both MI and MII cases with the cross-over distribution.

\section{Non-disjunction mapping}

Non-disjunction maps were constructed for the maternal MI and MII data using the map + program (31). Heterozygous loci in the parent contributing the additional chromosome were classified in the trisomic proband as either as ' $\mathrm{N}$ ' (not reduced to homozygosity) or ' $\mathrm{R}$ ' (reduced to homozygosity). In this way, transitions between reduced and non-reduced states could be reliably determined given sufficient coverage by informative markers. For non-disjunction mapping, we did not use cases with fewer than seven markers informative (Table 4). The theory for the analysis of tetrads follows Shahar and Morton (32) and is implemented in the map + program (11). Using map + , we computed the interference parameter $\mathrm{p}$ in the Rao function for the standard female map of chromosome 13 as 0.23 , corresponding to a relatively high level of chiasma interference (33). The truncation parameter $\mathrm{T}$, beyond which pairs of loci are separated by more than one recombination value (11), was computed at $55 \mathrm{cM}$ given the estimated level of chiasma interference. Maternal MI and MII non-disjunction maps were constructed using the data for which meiotic stage and number of transitions could be reliably determined.

\section{Comparison of non-disjunction and standard female genetic maps}

We examined and compared patterns of maternal recombination in a standard genetic map with non-disjunction maps constructed from the informative MI cases $(N=30)$ and MII cases $(N=32)$ (Table 2). There were insufficient paternal cases across both samples to construct non-disjunction maps.

Standard map. The standard female linkage map of chromosome 13 was constructed from the data from Icelandic families collected by Kong et al. (34).This comprised a total of 21 polymorphic markers typed and ordered by physical location in build 35 of the human genome sequence between $17308.183 \mathrm{~kb}$ (HECH1) and $110743.481 \mathrm{~kb}$ (D13S285). The standard female map spans $156.04 \mathrm{cM}$ (Fig. 1). Where genetic locations were not known directly, they were inferred by interpolation between genetic map flanking markers and using known physical locations.

\section{ACKNOWLEDGEMENTS}

This study was initiated by Professor Margareta Mikkelsen, the John F. Kennedy Institute, Glostrup, Denmark. Professor Mikkelsen had collected samples from some probands with trisomy 13 before the start and encouraged and supported the study. This study was supported by grants from Direktoer Jacob Madsens og Hustru Olga Madsens Fond, Direktoer Ib Henriksens Fond, Kong Christian den Tiendes Fond, Broedrene Hartmanns Fond, Fonden til Laegevidenskabens Fremme, Fru Lily Benthine Lunds Fond. The technicians at the chromosome and DNA laboratories at the Wilhelm Johannsen Centre and at the John F. Kennedy Institute are acknowledged for excellent work. Special thanks to Minna Becker, Anita Niebuhr, Kirsten Winther and Annemette Friis Mikkelsen. We are grateful to all the families, who accepted to participate in the study and gave samples, and all the clinical physicians, who collected the samples. The Wilhelm Johannsen Centre for Functional Genome Research was established by the Danish National Research Foundation.

Conflict of Interest statement. None declared.

\section{REFERENCES}

1. Patau, K., Smith, D.W., Therman, E., Inhorn, S.L. and Wagner, H.P. (1960) Multiple congenital anomaly caused by an extra autosome. Lancet, i, $790-793$.

2. de Grouchy, J. and Turleau, C. (1982) Atlas des maladies chromosomiques. Deuxième édition. Expansion Scientifique Francaise, Paris, pp. 228-234. 
3. Goldstein, H. and Nielsen, K.G. (1988) Rates and survival of individuals with trisomy 13 and 18. Data from a 10-year period in Denmark. Clin. Genet., 34, 366-372.

4. Jacobs, P.A., Hassold, T.J., Henry, H., Pettay, D. and Takaesu, N. (1987) Trisomy 13 ascertained in a survey of spontaneous abortions. J. Med. Genet., 24, 721-724.

5. Hassold, T., Jacobs, P.A., Leppert, M. and Sheldon, M. (1987) Cytogenetic and molecular studies of trisomy 13. J. Med. Genet., 24, $725-732$.

6. Zaragoza, M.V., Jacobs, P.A., James, R.S., Rogan, P., Sherman, S. and Hassold, T. (1994) Nondisjunction of human acrocentric chromosomes: studies of 432 trisomic fetuses and liveborns. Hum. Genet., 94, 411-417.

7. Robinson, W.P., Bernasconi, F., Dutly, F., Lefort, G., Romain, D.R., Binkert, F. and Schinzel, A.A. (1996) Molecular studies of translocations and trisomy involving chromosome 13. Am. J. Med. Genet., 61, 158-163.

8. Vital Statistics 2000. Statistics Denmark 2001. Copenhagen, Denmark.

9. Hassold, T., Merrill, M., Adkins, K., Freeman, S. and Sherman, S. (1995) Recombination and Maternal age-dependent nondisjunction: Molecular studies of trisomy 16. Am. J. Hum. Genet., 57, 867-874.

10. Petersen, M.B. and Mikkelsen, M. (2000) Nondisjunction in trisomy 21: origin and mechanisms. Cytogenet. Cell Genet., 91, 199-203.

11. Bugge, M., Collins, A., Petersen, M.B., Fisher, J., Brandt, C.A., Hertz, J.M., Tranebjærg, L., deLozier-Blanchet, C., Nicolaides, P., Brøndum-Nielsen, K. et al. (1998) Non-disjunction of chromosome 18 Hum. Mol. Genet., 7, 661-669.

12. Lamb, N.E., Freeman, S.B., Savage-Austin, A., Pettay, D., Taft, L., Hersey, J., Gu, Y., Shen, J., Saker, D., May, K.M. et al. (1996) Susceptible chiasmate configurations of chromosome 21 predispose to non-disjunction in both maternal meiosis I and meiosis II. Nature Genet., 14, 400-405.

13. Mikkelsen, M., Hallberg, A., Poulsen, H., Frantzen, M., Hansen, J. and Petersen, M.B. (1995) Epidemiological study of Down's syndrome in Denmark, including family studies of chromosome and DNA markers. Dev. Brain Dysfunct., 8, 4-12.

14. MacDonald, M., Hassold, T., Harvey, J., Wang, L.H., Morton, N.E. and Jacobs, P. (1994) The origin of 47,XXY and 47,XXX aneuploidy: heterogenous mechanisms and the role of aberrant recombination. Hum. Mol. Genet., 3, 1365-1371.

15. Petersen, M.B., Antonarakis, S.E., Hassold, T.J., Freeman, S.B., Sherman, S.L., Avramopoulos, D. and Mikkelsen, M. (1993) Paternal nondisjunction in trisomy 21: excess of male patients. Hum. Mol. Genet., 2, 1691-1695.

16. Tease, C., Geraldine, M., Hartshorne, M. and Hulten, M.A. (2002) Patterns of meiotic recombination in human fetal oocytes. Am. J. Hum. Genet., 70, 1469-1479.

17. Robinson, W.P., Bernasconi, F., Mutirangura, A., Ledbetter, D.H., Langlois, S., Malcolm, S., Morris, M.A. and Schinzel, A.A. (1993) Nondisjunction of chromosome 15:origin and recombination. Am. J. Hum. Genet., 53, 740-751.

18. Sherman, S.L., Petersen, M.B., Freeman, S.B., Hersey, J., Pettay, D., Taft, L., Frantzen, M., Mikkelsen, M. and Hassold, T.J. (1994) Nondisjunction of chromosome 21 in maternal meiosis I: evidence for maternal age-dependent nondisjunction mechanism involving reduced recombination. Hum. Mol. Genet., 3, 1529-1535.

19. Robinson, W.P., Kushinka, B.D., Petersen, M.B., Schultze, A., Brøndum-Nielsen, K., Christian, S.L., Ledbetter, D.H., Schinzel, A.A.,
Horsthemke, B., Schuffenbauer, S. et al. (1998) Maternal meiosis I non-disjunction of chromosome 15: dependence of the maternal age effect on level of recombination. Hum. Mol. Genet., 7, 1011-1019.

20. Petersen, M.B., Frantzen, M., Antonarakis, S.E., Warren, A.C., van Broeckhoven, C., Chakravarti, A., Cox, T.K., Lund, C., Olsen, B., Poulsen, H. et al. (1992) Comparative study of microsatellite and cytogenetic markers for detecting the origin of the nondisjoined chromosome 21 in Down syndrome. Am. J. Hum. Genet., 51, 516-525.

21. Hudson, T.J., Engelstein, M., Lee, M.K., Ho, E.C., Rubenfield, M.J., Adams, C.P., Housman, D.E. and Dracopoli, N.C. (1992) Isolation and chromosomal assignment of 100 highly informative human simple sequence repeat polymorphisms. Genomics, 13, 622-629.

22. Weissenbach, J., Gyapay, G., Dib, C., Vignal, A., Morisette, J., Millasseau, P., Vaysseix, G. and Lathrop, M. (1992) A second-generation linkage map of the human genome. Nature, 359, 794-801.

23. Weber, J., Kwitek, A. and May, P. (1990) Dinucleotide repeat polymorphism at the D13S71 locus. Nucleic Acids Res., 18, 4638.

24. Bowcock, A., Osborne-Lawrence, S., Barnes, R., Chakravarti, A., Washington, S. and Dunn, C. (1993) Microsatellite polymorphism linkage map of human chromosome 13q. Genomics, 15, 176-186.

25. Dib, C., Fauré, S., Fizames, C., Samson, D., Drouot, N., Vignal, A., Millasseau, P., Marc, S., Hazan, J., Seboun, E. et al. (1996) A comprehensive genetic map of the human genome based on 5,264 microsatellites. Nature., 380, 152-154.

26. Polymeropoulos, M.H., Rath, D.S., Xiao, H. and Merril, C.R. (1991) Dinucleotide repeat polymorphism at the human fms-related tyrosine kinase gene (FLT1). Nucleic Acids Res., 19, 2803.

27. Petersen, M.B., Schinzel, A.A., Binkert, F., Tranebjaerg, L., Mikkelsen, M., Collins, F.A., Economou, E.P. and Antonarakis, S.E. (1991) Use of short sequence repeat DNA polymorphisms after PCR amplification to detect the parental origin of the additional chromosome 21 in Down syndrome. Am. J. Hum. Genet., 48, 65-71.

28. Chakravarti, A. and Slaugenhaupt, S.A. (1987) Methods for studying recombination on chromosomes that undergo nondisjunction. Genomics, $1,35-42$.

29. Chakravarti, A. (1989) The probability of detecting the origin of nondisjunction of autosomal trisomies. Am. J. Hum. Genet., 44, 639-645.

30. Antonarakis, S.E., Avramopoulos, D., Blouin, J.L., Talbot, C.C. Jr and Schinzel, A.A. (1993) Mitotic errors in somatic cells cause trisomy 21 in $\sim 4.5 \%$ of cases and are not associated with advanced maternal age. Nature Genet., 3, 146-150.

31. Collins, A., Teague, J., Keats, B.J. and Morton, N.E. (1996) Linkage map integration. Genomics, 36, 157-162.

32. Shahar, S. and Morton, N.E. (1986) Origin of teratomas and twins. Hum. Genet., 74, 215-218.

33. Rao, D.C., Morton, N.E., Lindsten, J., Hultén, M. and Yee, S. (1977) A mapping function for man. Hum. Hered., 27, 99-104.

34. Kong, A., Gudbjartsson, D.F., Sainz, J., Jonsdottir, G.M., Gudjonsson, S.A., Richardsson, B., Sigurdardottir, S., Barnard, J., Hallbeck, B., Masson, G. et al. (2002) A high-resolution recombination map of the human genome. Nat. Genet., 31, 241-247.

35. Eggermann, T., Nöthen, M.M., Eiben, B., Hofmann, D., Hinkel, K., Fimmers, R. and Schwanitz, G. (1996) Trisomy of human chromosome 18: molecular studies on parental origin and cell stage of nondisjunction. Hum. Genet., 97, 218-223. 